GEOMETRY IN NONLINEAR CONTROL

AND DIFFERENTIAL INCLUSIONS

BANACH CENTER PUBLICATIONS, VOLUME 32

INSTITUTE OF MATHEMATICS

POLISH ACADEMY OF SCIENCES

WARSZAWA 1995

\title{
ASYMPTOTIC NULL CONTROLLABILITY OF BILINEAR SYSTEMS
}

\author{
FRITZ COLONIUS \\ Institut für Mathematik, Universität Augsburg \\ 86135 Augsburg, Germany \\ WOLFGANG KLIEMANN \\ Department of Mathematics, Iowa State University \\ Ames, Iowa 50011, U.S.A.
}

\begin{abstract}
The region of asymptotic null controllability of bilinear systems with control constraints is characterized using Lyapunov exponents. It is given by the cone over the region of attraction of the maximal control set in projective space containing zero in its spectral interval.
\end{abstract}

1. Introduction. In this paper we use recent results on the Lyapunov spectrum of families of time varying matrices [11] in order to characterize the domain of null controllability of bilinear systems with constrained control range. Our approach is based on the classical concept of Lyapunov exponents and on their approximation by Floquet exponents. Lyapunov exponents were introduced by A. V. Lyapunov in his 1892 thesis [16] (as "order numbers") as a tool to study nonlinear differential equations via their linearizations along trajectories. This is Lyapunov's first method as opposed to his second, direct method, which is now known as the concept of Lyapunov functions and which is widely employed in control theory. Lyapunov exponents have a rich history in deterministic and stochastic dynamics (see e.g. [3], [12], [17] and the proceedings [1, 2]), but to our knowledge they have not been used systematically in control theory. We hope that the results of the present paper will encourage research in this direction.

In particular, the study of nonlinear control systems with a common fixed point $x^{*}$ can profit from the detailed analysis of the family of equations obtained by linearizing in $x^{*}$ with respect to $x$ only. This gives the following bilinear control

1991 Mathematics Subject Classification: 93D05, 93B05.

Partially supported by DFG grant no. Co124/8-2 and by ONR grant no. N00014-93-1-0868.

The paper is in final form and no version of it will be published elsewhere. 
systems, which are discussed in the present paper:

$$
\begin{gathered}
\frac{d}{d t} x(t)=A_{0} x(t)+\sum_{i=1}^{m} u_{i}(t) A_{i} x(t)=: A(u(t)) x(t) \quad \text { in } \mathbf{R}^{d}, \\
\mathrm{u} \in \mathcal{U}=\left\{u: \mathbf{R} \rightarrow \mathbf{R}^{m} \mid u(t) \in U \text { a.e. }\right\} .
\end{gathered}
$$

Here $A_{0}, A_{1}, \ldots, A_{m}$ are $d \times d$-matrices and $U \subset \mathbf{R}^{m}$ is compact and convex with $0 \in$ int $U$, the interior of $U$.

The solution of (1) corresponding to $u \in \mathcal{U}$ and initial value $0 \neq x \in \mathbf{R}^{d}$ will be denoted by $\phi(t, x, u), t \in \mathbf{R}$. For a given control function $u \in \mathcal{U},(1)$ is a linear differential equation with time varying coefficients. The exponential growth behavior of a solution is measured by the corresponding Lyapunov exponent

$$
\lambda(u, x)=\limsup _{t \rightarrow \infty} \frac{1}{t} \log |\phi(t, x, u)|
$$

where $|\cdot|$ denotes the Euclidean norm in $\mathbf{R}^{d}$ (since all norms in $\mathbf{R}^{d}$ are equivalent, any norm will yield the same Lyapunov exponent). Classical properties of Lyapunov exponents are described, e.g., in [14], Chapter 8, or in [4]. Here we only remark that for constant $A(u)$, the Lyapunov exponents $\lambda(u, x), x \neq 0$, are the real parts of the eigenvalues of $A(u)$; for periodic $A(u)$, they are the corresponding Floquet exponents. In general, the limit in (3) need not exist. This question is closely related to the (difficult) problem of Lyapunov regularity. In any case, $\lambda(u, x)<0$ iff $\phi(t, x, u)$ converges to zero faster than any exponential $\exp (a t), \lambda(u, x)<a<0$ (and slower than those with $a<\lambda(u, x)$ ). However, note that-for fixed general $u-\lambda(u, x)<0$ for all $x \in \mathbf{R}^{d} \backslash\{0\}$ does imply neither exponential stability nor uniform stability (see [14], Section 65).

We will characterize the region of asymptotic null controllability, i.e.

$$
\mathcal{N}=\left\{0 \neq x \in \mathbf{R}^{d} \mid \text { there exists } u \in \mathcal{U} \text { with } \lambda(u, x)<0\right\} .
$$

A moment of reflection on the uncontrolled equation (1) with $\mathcal{U}=\{0\}$ reveals that in this case the eigenvalues and the eigenspaces determine the "stabilizability" properties. Similarly, if $A(t)$ is a time varying matrix function such that there is at least one negative Lyapunov exponent for $\dot{x}=A(t) x$, then there exists a lower dimensional subvariety $N$ of $\mathbf{R}^{d}$ such that the solutions with initial values in $N$ tend to zero for $t \rightarrow \infty$, compare [16]. The theory developed below is built in analogy to these ideas, using appropriate generalized versions of eigenspaces associated with Lyapunov exponents. It turns out that for systems of the form (1) the regions of asymptotic null controllability have nonvoid interior and are characterized by controllability properties of the projected system on the projective space in $\mathbf{R}^{d}$.

In Section 2, we collect the main results from [11] on the Lyapunov spectrum and its relation to the control sets in projective space. In Section 3, this is used for a characterization of the region $\mathcal{N}$ of asymptotic null controllability. We also show, how one can - in the two dimensional case - construct from this analysis 
stabilizing feedbacks. Finally, we characterize the control sets in affine space $\mathbf{R}^{d}$ as cones corresponding to the control sets in projective space with corresponding spectral interval containing zero in its interior.

2. Spectral theory of families of time varying matrices. In this section, we cite results on the approximation of the Lyapunov spectrum by Floquet exponents of periodic matrix functions corresponding to admissible controls $u \in \mathcal{U}$. The proofs in [11] also make essential use of methods from the theory of flows on vector bundles, in particular, the Morse spectrum (cp. [10]). Here we concentrate on the results only as far as they are relevant for the control theoretic applications treated in this paper.

The exponential growth behavior of linear differential equations can be studied via the associated (angular) system on projective space $\mathbf{P}^{d-1}$ obtained by identifying opposite points on the sphere in $\mathbf{R}^{d}$ :

The angular component of (1) is

$$
\frac{d}{d t} s(t)=h_{0}(s(t))+\sum_{i=1}^{m} u_{i}(t) h_{i}(s(t))=: h(u(t), s(t)) \quad \text { on } \mathbf{P}^{d-1}
$$

where $h_{j}(s):=\left(A_{j}-s^{T} A_{j} s \cdot I\right) s$ for $j=0, \ldots, m$. The superscript ${ }^{T}$ denotes the transpose.

Solving the corresponding equation for the radial component yields for the Lyapunov exponent

$$
\lambda(u, x)=\limsup _{t \rightarrow \infty} \frac{1}{t} \int_{0}^{t} q(u(\tau), s(\tau)) d \tau
$$

with $q(u, s)=q_{0}(s)+\sum_{i=1}^{m} u_{i} q_{i}(s), q_{j}(s)=s^{T} A_{j} s$, and $s_{0}=x /|x|$, the projection of $x \in \mathbf{R}^{d} \backslash\{0\}$ onto $\mathbf{P}^{d-1}$; here $s(\tau)=\phi\left(\tau, s_{0}, u\right)$ denotes the solution of (5) with $\phi\left(0, s_{0}, u\right)=s_{0} \in \mathbf{P}^{d-1}$. Thus the Lyapunov exponents only depend on the behavior of the nonlinear system (5) on projective space. In order to analyze this (analytic) control system, we assume local accessibility, which is equivalent to the following Lie algebra rank condition:

$$
\operatorname{dim} \mathcal{L} \mathcal{A}\{h(\cdot, u) \mid u \in U\}(x)=d-1 \quad \text { for all } x \in \mathbf{P}^{d-1} .
$$

It turns out that the control sets of (5) are closely related to the (generalized) eigenspaces of the elements in the systems semigroup

$$
\begin{array}{r}
\mathcal{S}=\left\{g=\exp \left(t_{n} B_{n}\right) \ldots \exp \left(t_{1} B_{1}\right) \mid t_{j} \geq 0, B_{j}=A\left(u^{j}\right), \text { for some } u^{j} \in U\right. \\
j=1, \ldots, n, n \in \mathbf{N}\} .
\end{array}
$$

Note that by $(\mathbf{H})$, the interior of $\mathcal{S}$ in the systems (Lie) group $\mathcal{G}$ (where the $t_{j}$ are arbitrary in $\mathbf{R}$ ) is nonvoid. Then the control structure of (5) is revealed by the following result (see [8], Theorem 3.10). 
1. TheOREM. Let $(\mathbf{H})$ be satisfied.

(i) There are $1 \leq k \leq d$ control sets $D_{1}, \ldots, D_{k}$ with nonvoid interior in $\mathbf{P}^{d-1}$, called the main control sets.

(ii) The interiors of the main control sets are the connected components of $\mathbf{P} V=\{\mathbf{P} E(\lambda) \mid \lambda \in \operatorname{spec}(g), g \in$ int $\mathcal{S}\}$, where $\mathbf{P} E(\lambda)$ denotes the generalized eigenspace of $g$ corresponding to $\lambda$, projected onto $\mathbf{P}^{d-1}$.

(iii) The main control sets are linearly ordered by

$$
D_{i} \prec D_{j} \text { if there exist } x_{i} \in D_{i}, x_{j} \in D_{j} \text {, and } g \in \mathcal{S}_{t} \text { with } g x_{i}=x_{j} \text {. }
$$

We enumerate the control sets such that $D_{1} \prec \ldots \prec D_{k}$.

This result leads us to the following definition.

2. Definition. Let $D$ be a main control set of (5). The Floquet spectrum of (1) over a main control set $D$ is defined as

$$
\begin{aligned}
\Sigma_{F l}(D)=\{\lambda(u, p) \mid & (u, p) \in \mathcal{U} \times \operatorname{int} D, u \text { is piecewise constant and } \\
& \tau \text {-periodic such that } \phi(\tau, p, u)=p \text { for some } \tau \geq 0\} .
\end{aligned}
$$

The Floquet spectrum $\Sigma_{F l}$ of (1) is

$$
\Sigma_{F l}=\bigcup_{i=1}^{k} \Sigma_{F l}\left(D_{i}\right) .
$$

Similarly, the Lyapunov spectrum of (1) over $\operatorname{cl}(D)$ (the closure of $D$ ) is

$$
\Sigma_{L y}(\operatorname{cl}(D))=\{\lambda(u, p) \mid \text { there exists } T \geq 0
$$

$$
\text { such that } \phi(t, p, u) \in \operatorname{cl}(D) \text { for all } t \geq T\}
$$

and the Lyapunov spectrum is

$$
\Sigma_{L y}=\left\{\lambda(u, p) \mid u \in U, p \in \mathbf{P}^{d-1}\right\} .
$$

Clearly, $\Sigma_{F l}(D) \subset \Sigma_{L y}(\operatorname{cl}(D)) \subset \Sigma_{L y}$. Concerning the converse direction, it is not known, in general, when equality holds. However, more can be said if we embed the system (1) into a family of systems depending on a parameter $\rho \geq 0$ indicating the size of the control range.

Let $U^{\rho}:=\rho U, \rho \geq 0$. Then all quantitities considered above depend on $\rho$. Note that $(\mathbf{H})$ holds for $\rho=1$, iff it holds for some $\rho>0$.

Let $\lambda_{1}, \ldots, \lambda_{k}$ be the different real parts of the eigenvalues of the matrix $A_{0}$, and let $E\left(\lambda_{i}\right), 1 \leq i \leq k \leq d$, be the corresponding sums of generalized eigenspaces. Consider for $i=1, \ldots k$ the following maps on $[0, \infty)$ with values in the compact subsets of $\mathbf{P}^{d-1}$, endowed with the Hausdorff metric:

$$
\rho \longmapsto \operatorname{cl}\left(D_{i}(\rho)\right),
$$

where $D_{i}(\rho)$ is the main control set with $\mathbf{P} E\left(\lambda_{i}\right) \subset$ int $D_{i}(\rho)$ for $\rho>0$ and $D_{i}(0)=\mathbf{P} E\left(\lambda_{i}\right)$.

Note that the number $k(\rho)$ of main control sets is decreasing. Hence some of the $D_{i}(\rho), i=1, \ldots, k$, in $(7)$ may coincide. We denote the different $D_{i}(\rho)$ 
by $D_{j}^{\rho}, j=1, \ldots, k(\rho)$. The following theorem from [11], Corollary 5.6, shows in particular, that main control sets with the properties indicated in (7) exist, i.e. the map is well defined.

3. Theorem. Assume that $(\mathbf{H})$ and the following $\rho-\rho^{\prime}$ inner pair condition (I) are satisfied:

(I) For all $\rho^{\prime}>\rho>0$ and all $(u, p) \in \mathcal{U}^{\rho} \times \mathbf{P}^{d-1}$ there exist $T>0$ and $S>0$ such that $\phi(T, p, u) \in$ int $\mathcal{O}_{\leq T+S}^{\rho^{\prime,+}}(p)$ where $\mathcal{O}_{\leq T+S}^{\rho^{\prime++}}(p)=\left\{q \in \mathbf{P}^{d-1} \mid\right.$ there are $v \in \mathcal{U}^{\rho^{\prime}}$ and $0<t \leq T+\bar{S}$ with $\phi(t, p, u)=q$ \}.

Then the following assertions hold:

1. For $i=1, \ldots, k$ and $\rho>0$ there is a main control set $D_{i}(\rho)$ satisfying the condition in $(7)$ and $\mathrm{cl} D_{i}(\rho) \subset$ int $D_{i}\left(\rho^{\prime}\right)$ for $\rho^{\prime}>\rho>0$.

2. The maps $\rho \longmapsto \mathrm{cl} \Sigma_{F l}\left(D_{i}(\rho)\right)$ are increasing, continuous outside at most countably many $\rho$-values in $[0, \infty)$, and the images are intervals. At the continuity points $\operatorname{cl} \Sigma_{F l}(D)=\Sigma_{L y}(c l(D)), i=1, \ldots, k$, and $\operatorname{cl} \Sigma_{F l}=\Sigma_{L y}$.

3. Let $\rho$ be a continuity point, then for each $(u, x) \in \mathcal{U}^{\rho} \times \mathbf{P}^{d-1}$ there is a main control set $D$ with $\pi_{\mathbf{P}} \omega(u, x):=\left\{q \in \mathbf{P}^{d-1} \mid\right.$ there are $t_{k} \rightarrow \infty$ with $\left.\phi\left(t_{k}, x, u\right) \rightarrow q\right\} \subset \operatorname{cl}(D)$ and $\lambda(u, x) \in \Sigma_{L y}(\operatorname{cl}(D))$.

4. At each continuity point $\rho$, define $E_{j}^{\rho}(u)=\left\{x \in \mathbf{R}^{d} \mid x \neq 0\right.$ implies $\phi(t, x, u) \in \operatorname{cl} D_{j}^{\rho}$ for $\left.t \in \mathbf{R}\right\}, j=1, \ldots, k(\rho), u \in \mathcal{U}$. Then the $E_{j}^{\rho}(u)$ are subspaces of dimension independent of $u \in \mathcal{U}$, such that

$$
\mathbf{R}^{d}=E_{1}^{\rho}(u) \oplus \ldots \oplus E_{k(\rho)}^{\rho}(u) .
$$

Rem ark. The spectral intervals are ordered via $\Sigma_{F l}\left(D_{i}^{\rho}\right) \prec \Sigma_{F l}\left(D_{j}^{\rho}\right)$ if $i<j$ in the following sense: $\inf \Sigma_{F l}\left(D_{i}^{\rho}\right)<\Sigma_{F l}\left(D_{j}^{\rho}\right)$ and $\sup \Sigma_{F l}\left(D_{i}^{\rho}\right)<\sup \Sigma_{F l}\left(D_{j}^{\rho}\right)$.

Remark. The spectral intervals $\mathrm{cl} \Sigma_{F l}\left(D_{i}^{\rho}\right)$ may overlap: see Example 3.2 in $[8]$.

3. Asymptotic null controllability. In this section we characterize the region $\mathcal{N}$ of asymptotic null controllability of bilinear systems. Furthermore, in the two dimensional case, one obtains from our analysis a stabilizing feedback, which is homogeneous. Finally, we characterize the control sets in $\mathbf{R}^{d}$.

Consider the following parametrized family of bilinear control systems depending on $\rho>0$ :

$$
\begin{aligned}
& \frac{d}{d t} x(t)=A_{0} x(t)+\sum_{i=1}^{m} u_{i}(t) A_{i} x(t) \text { in } \mathbf{R}^{d}, \\
& u \in \mathcal{U}:=\left\{u: \mathbf{R} \rightarrow U^{\rho}:=\rho \cdot U \mid \text { measurable }\right\},
\end{aligned}
$$

where $A_{0}, \ldots, A_{k}$ are $d \times d$ matrices and $U \subset \mathbf{R}^{m}$ is compact and convex with $0 \in$ int $U$. 
Throughout this section, we assume that $(\mathbf{H})$ and $(\mathbf{I})$ hold. For a main control set $D \subset \mathbf{P}^{d-1}$ of the projected system we denote its domain of attraction by

(9) $\mathcal{A}(D):=\left\{q \in \mathbf{P}^{d-1} \mid\right.$ there is $u \in \mathcal{U}$ and $t \geq 0$ with $\left.\phi(t, q, u) \in D\right\}$

where $\phi(t, q, u)$ denotes again the solution of (5). Recall the linear ordering of the main control sets and let $s(\rho):=\max \left\{i \in\{1, \ldots, k(\rho)\} \mid \inf \Sigma_{F l}\left(D_{i}^{\rho}\right)<0\right\}$.

4. Theorem. Assume that conditions $(\mathbf{H})$ and $(\mathbf{I})$ hold for (8).

(i) For all $\rho>0$ and all $\epsilon>0$ there exist continuity points $\rho_{1}, \rho_{2}$ of the spectral intervals with $\rho-\epsilon<\rho_{1}<\rho<\rho_{2}<\rho+\epsilon$; here cl $\Sigma_{F l}\left(D_{i}^{\rho_{j}}\right)=\Sigma_{L y}\left(\operatorname{cl}\left(D_{i}^{\rho_{j}}\right)\right)$, $i=1, \ldots, k(\rho), j=1,2$.

(ii) Let $\rho$ be a continuity point of the spectral intervals. Then $x \in \mathbf{R}^{d} \backslash\{0\}$ is in the region $\mathcal{N}^{\rho}$ of asymptotic null controllability if $x \in \mathcal{A}^{\rho}\left(D_{s(\rho)}^{\rho}\right)$. Conversely, if $x \in \mathbf{R}^{d} \backslash\{0\}$ is in the region of asymptotic null controllability, then $x \in \mathcal{A}^{\rho}\left(D_{s(\rho)}^{\rho}\right)$ or, with $p=\pi_{\mathbf{P}} x$,

$$
\begin{array}{r}
\text { there is } u \in \mathcal{U}^{\rho} \text { with } \lambda(u, x)<0 \text { and } \pi_{\mathbf{P}} \omega(u, p) \subset \operatorname{cl} D_{i}^{\rho} \backslash D_{i}^{\rho} \\
\text { for some } i \leq s(\rho) .
\end{array}
$$

(iii) If $(u, x) \in \mathcal{U}^{\rho} \times \mathbf{P}^{d-1}$ satisfies (10), then $u \in \mathcal{U}^{\rho} \backslash \mathcal{U}^{\rho^{\prime}}$ for all $\rho^{\prime}<\rho$ and $x \in \mathcal{A}^{\rho}\left(D_{s(\rho)}^{\rho}\right)$ for all $\rho^{\prime}>\rho$.

Remark. Theorem 3 above shows in particular that typically $\rho$ will be a continuity point of the spectral intervals (for example, the Lebesgue measure of all points in $[0, \infty)$, which are not a continuity points, is zero). By Theorem 4 with the exception of the pairs $(u, x)$ satisfying (10), the region of asymptotic null controllability is given by the domain of attraction of the control set $D_{s(\rho)}^{\rho}$. Finally, Property (10) is shown to be exceptional.

Proof of Theorem 4. (i) This is immediate from Theorem 3.

(ii) Abbreviate $s=s(\rho)$ and let $x \in \mathcal{A}^{\rho}\left(D_{s(\rho)}^{\rho}\right)$. Then there exist $(u, q) \in$ $\mathcal{U}^{\rho} \times \mathbf{P}^{d-1}$ such that $(u(\cdot), \phi(\cdot, q, u))$ is periodic with $\phi(t, q, u) \in$ int $D_{s}^{\rho}$ for all $t \in \mathbf{R}$, and $\lambda(u, q)<0$. Since $p:=\pi_{\mathbf{P}} x \in \mathcal{A}^{\rho}\left(D_{s}^{\rho}\right)$ there are $v \in \mathcal{U}^{\rho}$ and $t_{0} \geq 0$ such that $q=\phi\left(t_{0}, p, u\right)$. The concatenation of $v$ and the periodic control $u$ (applied after $t_{0}$ ) controls $x$ asymptotically to the origin. Conversely, suppose that there is $u \in \mathcal{U}^{\rho}$ with $\lambda(u, x)<0$. By Theorem 3 it follows that there is a control set $D_{i}^{\rho}$ with $\pi_{\mathbf{P}} \omega(u, p) \subset \operatorname{cl}\left(D_{i}^{\rho}\right)$. Hence

$$
\lambda(u, x) \in \operatorname{cl} \Sigma_{F l}\left(D_{i}^{\rho}\right)=\Sigma_{L y}\left(\operatorname{cl}\left(D_{i}^{\rho}\right)\right) .
$$

If $\pi_{\mathbf{P}} \omega(u, p) \subset D_{i}^{\rho}$, for some $i$, then by Theorem 3 it follows that $i \leq s$, and $p$ can be steered to $D_{s}^{\rho}$, by Theorem 1, i.e. $p \in \mathcal{A}^{\rho}\left(D_{s}^{\rho}\right)$. Otherwise, (10) holds.

(iii) This follows from Theorem 3 , since the control sets are strictly increasing.

Remarks. (a) The set of exceptional pairs $(u, x)$ satisfying (10) can be nonvoid; their behavior has to be studied separately, see Example 6, below. 
(b) The proof of the theorem above shows that - except for (10) - in case of asymptotic null controllability from $x$ the control $u \in \mathcal{U}$ can be chosen in such a way that there exists $T \geq 0$ such that after time $T, u$ is periodic, and $\phi(t, x, u)$ is periodic in $\mathbf{P}^{d-1}$ with the same period. Periodicity of $u$ (i.e. $T=0$ ) is possible if $\pi_{\mathbf{P}} x \in$ int $D$ for some control set $D_{i}$ with $i \leq s(\rho)$.

The interest in asymptotic null controllability stems, among other reasons, from the fact that this is a necessary condition for feedback stabilization at the origin via static state feedback, compare e.g. [18]. The question whether a stabilizing feedback exists depends, of course, on the class of admissible feedbacks:

- For constant feedbacks $u(x) \equiv u$ the question reduces to that of the constant spectrum of (8) and the corresponding eigenspaces.

- For time varying feedback $u(t, x) \equiv u(t)$ one has to consider (for stabilization from all $\left.x \in \mathbf{R}^{d} \backslash\{0\}\right)$ the quantity $\inf _{g \in \text { int } \mathcal{S}_{t}} \max _{\lambda \in \operatorname{spec} g} \frac{1}{t} \log |\lambda|$, see [7].

Here we allow nonlinear, piecewise continuous and a-priori bounded feedback: The system (8) is feedback stabilizable at the origin from $x \in \mathbf{R}^{d} \backslash\{0\}$ if there exists a piecewise continuous function $u: K \rightarrow U$ defined on a cone $K \subset \mathbf{R}^{d} \backslash\{0\}$ containing $x$ such that:

(11) The solutions $\phi\left(t, x^{0}, u\right)$ of (8) with $\phi\left(0, x^{0}, u\right)=x^{0}$ are well defined for all $x^{0} \in K, \phi\left(t, x^{0}, u\right) \in K$ for all $t \geq 0$, and $\lambda(u, x)<0$.

The stabilization problem (11) has a complete solution for two dimensional systems, see also [5], who use an entirely different method (Lyapunov functions), in the unconstrained case.

5. Theorem. Consider the bilinear control system (8) for $d=2$ and assume $(\mathbf{H})$. The system is feedback stabilizable at the origin from $x \in \mathbf{R}^{d} \backslash\{0\}$ in the sense of (11) iff $x$ is asymptotically null controllable.

Pr o of. Let $x \in \mathbf{R}^{d} \backslash\{0\}$ be asymptotically null controllable. It follows from Theorem 4.1 in [15] (cp.also [11], Corollary 4.9), that only the following cases can occur for $d=2$ :

- (a) inf $\Sigma_{F l}\left(D_{s}\right)$ is a real eigenvalue for a constant matrix $A=A_{0}+\sum_{i=1}^{m} u_{i} A_{i}$ for some $u \in U$ such that there is $\lambda \in \operatorname{spec} A$ with $\mathbf{P} E(\lambda) \subset$ int $D_{s}$.

(aa) There are two main control sets and $s=1$ : in this case we have either $p=x /|x| \in$ int $D_{1}$ or $p \in \partial D_{1}$. In the first case, we parametrize $D_{1}$ via the angle as $\left(\theta_{1}, \theta_{2}\right) \subset[0, \pi)$. Then $\mathbf{P} E(\lambda) \in\left(\theta_{1}, \theta_{2}\right)$ and there exist constant matrices $B_{1}$ and $B_{2}$ with real eigenvalues $\mu_{1}$ and $\mu_{2}$, respectively, such that $\mathbf{P} E\left(\mu_{1}\right)=\theta_{1}$, $\mathbf{P} E\left(\mu_{2}\right)=\theta_{2}$. Define a piecewise constant feedback $u(\theta)$ on $\operatorname{cl}\left(D_{1}\right)$ by

$$
u(\theta)= \begin{cases}B_{1} & \text { for } \theta \in\left[\theta_{1}, \mathbf{P} E(\lambda)\right), \\ A & \text { for } \theta=\mathbf{P} E(\lambda), \\ B_{2} & \text { for } \theta \in\left(\mathbf{P} E(\lambda), \theta_{2}\right),\end{cases}
$$

which stabilizes the system for all $\theta \in D_{1}$. 
If $p \in \partial D_{1}$, then the system is asymptotically null controllable from $x$ iff $p=\mathbf{P} E(\lambda)$ for some $\lambda \in \operatorname{spec} B$ with $B$ constant and $\lambda<0$. Here we use the feedback $B$.

(ab) $s=2$ : In this case we can set $u(x)=u \in U$ with $A_{0}+\sum_{i=1}^{m} u_{i} A_{i}=A$.

(ac) There is only one main control set: In this case we can find a piecewise constant feedback $u(p): \mathbf{P}^{1} \rightarrow U$ that stabilizes the projected system at $\mathbf{P} E(\lambda)$, compare [9]. This feedback stabilizes the system (8) in the sense of (11).

- (b) inf $\Sigma_{F l}\left(D_{s}\right)$ is not a real eigenvalue of any constant matrix. In this case, we have only one main control set $D=\mathbf{P}^{1}$, and we can find a piecewise constant $u^{0} \in \mathcal{U}$ and $p^{0} \in \mathbf{P}^{1}$ such that $\lambda\left(u^{0}, p^{0}\right)<0,\left(u^{0}\left(t^{0}\right), \phi\left(t^{0}, p^{0}, u^{0}(\cdot)\right)\right)=\left(u^{0}(0), p^{0}\right)$ for some $t^{0}>0$ with $t^{0}=\min \left\{t>0 \mid \phi\left(t, p^{0}, u^{0}(\cdot)\right)=p^{0}\right\}$, and $\left(u^{0}, \phi\left(\cdot, p^{0}, u^{0}(\cdot)\right)\right.$ is $t^{0}$-periodic without loops. For $p \in \mathbf{P}^{1}$ set $u(p)=u^{0}$ if $p=\phi\left(t, p^{0}, u^{0}(\cdot)\right)$ for some $t \in\left[0, t^{0}\right)$. This feedback $u: \mathbf{P}^{1} \rightarrow U$ is well defined and stabilizes the system in the sense of (11).

The following example illustrates some of the features occuring above, in particular the role of the exceptional points satisfying (10).

6. ExAmple. Consider the linear oscillator

$$
\frac{d}{d t} x(t)=\left(\begin{array}{cc}
0 & 1 \\
-1 & -2 b
\end{array}\right) x(t)+u(t)\left(\begin{array}{rr}
0 & 0 \\
-1 & 0
\end{array}\right) x(t)
$$

with $u(t) \in U=[-2,2]$ and $b=-2$. Here we have two control sets $D_{1}$ and $D_{2}$ with spectral intervals $\Sigma_{F l}\left(D_{1}\right)=(2-\sqrt{5}, 1)$ and $\Sigma_{F l}\left(D_{2}\right)=(3,2+\sqrt{5})$. The set $D_{1}$ is given by

$$
D_{1}=\pi_{\mathbf{P}}\left\{\left(\begin{array}{l}
x_{1} \\
x_{2}
\end{array}\right) \in \mathbf{R}^{2} \backslash\{0\} \mid x_{2}=\alpha x_{1}, \alpha \in(2-\sqrt{5}, 1)\right\} .
$$

At the boundary point $\pi_{\mathbf{P}}\left(\begin{array}{c}x_{1} \\ (2-\sqrt{5}) x_{1}\end{array}\right)$ the Lyapunov exponent is $2-\sqrt{5}<0$, while at $\pi_{\mathbf{P}}\left(\begin{array}{l}x_{1} \\ x_{1}\end{array}\right)$ the exponent is $1>0$. Hence the maximal cone of feedback stabilization is in this case

$$
K=\left\{\left(\begin{array}{l}
x_{1} \\
x_{2}
\end{array}\right) \in \mathbf{R}^{2} \backslash\{0\} \mid x_{2}=\alpha x_{1}, \alpha \in[2-\sqrt{5}, 1)\right\} .
$$

This example with $U=[-1,1]$ and $b=\frac{1}{4}$ shows also that the constant spectrum may be strictly contained in the Floquet spectrum and that the stabilizing feedback may need at least two values, cp. [7], Section 6.

Re marks. (a) The set of points from which a system is feedback stabilizable at the origin can be a nontrivial subset of $\mathbf{R}^{d}$, as the example above shows.

(b) If one is interested in feedback stabilization from all $x \neq 0$, i.e. $K=\mathbf{R}^{d}$, then no exceptional boundary points have to be considered, because each point in $\mathbf{P}^{d-1}$ can be controlled into the interior of the maximal control set int $D_{k}$. 
We now turn to the controllability problem for bilinear control systems of the form (8). We note that the present approach is different from the one that is, e.g., used in [13]. A necessary condition for controllability is accessibility, which for analytic systems is equivalent to the Lie algebra rank condition. Hence we will assume from now on the following condition $\left(\mathbf{H}_{\mathrm{aff}}\right)$ :

$\left(\mathbf{H}_{\mathrm{aff}}\right)$

$$
\operatorname{dim} \mathcal{L} \mathcal{A}\left\{A_{0}+\sum_{i=1}^{m} u_{i} A_{i} \mid u \in U\right\}(x)=d \quad \text { for all } x \in \mathbf{R}^{d} \backslash\{0\} .
$$

Note that $\left(\mathbf{H}_{\text {aff }}\right)$ implies $(\mathbf{H})$ for the projected system.

It is clear that the system (8) cannot be completely controllable outside of the cones in $\mathbf{R}^{d}$ generated by the control sets of the projected system. Note that there are at most $2 d$ cones in $\mathbf{R}^{d}$, corresponding to the main control sets in $\mathbf{P}^{d-1}$. Using spectral theory for the family of matrices $\left\{A_{0}+\sum_{i=1}^{m} u_{i} A_{i} \mid u \in U\right\}$ we obtain for the cones over the main control sets:

7. Theorem. Assume that $\left(\mathbf{H}_{\mathrm{aff}}\right)$ holds, and let $D$ be a main control set of the projected system.

1. If $0 \in$ int $\Sigma_{F l}(D)$, then the system (8) is completely controllable in the cone $K:=\{\alpha p \mid p \in$ int $D, \alpha>0\}$. by $D$.

2. If $0 \notin \Sigma_{L y}(\mathrm{cl}(D))$, then the system is not controllable in the cone generated

P r o of. The second assertion is obvious and it only remains to show the first one:

Let $(u, p) \in \mathcal{U} \times$ int $D$ be such that $\lambda(u, p)=0$ and $p \in \mathbf{P} E(0)$ for $g_{u} \in$ int $\mathcal{S}$. Then there exist $T>0$ and $\delta>0$ such that for all $\lambda \in(-\delta, \delta)$ there is a piecewise constant $u \in \mathcal{U}$ with $\lambda \in \operatorname{spec} g_{u}, g_{u} \in$ int $\mathcal{S}_{\leq T}$ and $\mathbf{P} E\left(\lambda, g_{u}\right) \subset N$, where $N$ is a compact neighborhood of $p$ with $N \subset$ int $D \subset \mathbf{P}^{d-1}$. Denote $L_{p}=\{\alpha p \mid \alpha>0\} \subset \mathbf{R}^{d}$ and let $[\alpha p, \beta p]$ be a compact interval on $L_{p}$. By uniform boundedness of the first hitting time in $N$ (see [6]) and the above, there exist piecewise constant $v_{i} \in \mathcal{U}, i=1,2$, such that $g_{v_{1}}(\alpha p)=\gamma_{1} p, g_{v 2}(\beta p)=\gamma_{2} p$ with $\gamma_{1}, \gamma_{2} \in(\alpha, \beta)$. Hence we obtain

$$
\bigcup_{n \geq 0} g_{v_{1}}^{n}[\alpha p, \beta p] \cup \bigcup_{n \geq 0} g_{v_{2}}^{n}[\alpha p, \beta p]=L_{p} .
$$

Now pick $x, y \in K$. Since $\pi_{\mathbf{P}} x \in$ int $D$, there exist $u_{1} \in \mathcal{U}$ and $t_{1} \geq 0$ such that the corresponding solution on $\mathbf{P}^{d-1}$ satisfies $\phi\left(t_{1}, \pi_{\mathbf{P}} x, u_{1}\right)=p$. Hence, by $\left(\mathbf{H}_{\text {aff }}\right)$, there is an interval $\left[\alpha_{1} p, \beta_{1} p\right] \subset L_{p}$ with $\left[\alpha_{1} p, \beta_{1} p\right] \subset$ int $\mathcal{O}^{+}(x)$. By a similar argument we have that there is an interval $\left[\alpha_{2} p, \beta_{2} p\right] \subset L_{p}$ with $\left[\alpha_{2} p, \beta_{2} p\right] \subset$ int $\mathcal{O}^{-}(y)$. Hence $y \in \mathcal{O}^{+}(x)$, which proves the assertion.

Re m a r k. If $\rho$ is a continuity point of the spectral intervals, then $\mathrm{cl} \Sigma_{F l}(D)=$ $\Sigma_{L y}(\operatorname{cl}(D))$, and the only case not covered by the theorem above is when $0 \in$ $\partial \Sigma_{L y}(\operatorname{cl}(D))$, the boundary of $\Sigma_{L y}(\operatorname{cl}(D))$. 


\section{References}

[1] L. Arnold, H. Crauel and J.-P. Eckmann (eds.), Lyapunov Exponents, Lecture Notes in Math. 1486, Springer, 1991.

[2] L. Arnold and V. Wihstutz, Lyapunov Exponents, Lecture Notes in Math. 1186, Springer, 1986.

[3] B. F. Bylov, R. E. Vinograd, D. M. Grobman, V. V. Nemytskiŭ, Theory of Lyapunov Exponents, Nauka, Moscow, 1966 (in Russian).

[4] L. Cesari, Asymptotic Behavior and Stability Problems in Ordinary Differential Equations, 3rd ed., Springer, 1971.

[5] R. Chabour, G. Sallet and J. C. Vivalda, Stabilization of nonlinear systems: a bilinear approach, Math. Control Syst. Signals (1993), to appear.

[6] F. Colonius and W. Kliemann, Infinite time optimal control and periodicity, Appl. Math. Optim. 20 (1989), 113-130.

[7] _-, - Maximal and minimal Lyapunov exponents of bilinear control, J. Differential Equations 101 (1993), 232-275.

[8] - - - Linear control semigroups acting on projective space, J. Dynamics Differential Equations 5 (1993), 495-528.

[9] - - - Controllability and stabilization of one dimensional systems, Systems Control Lett. 24 (1995), 87-95.

[10] _ - - The Morse spectrum of linear flows on vector bundles, Trans. Amer. Math. Soc. (1995), to appear.

[11] - - - The Lyapunov spectrum of families of time-varying matrices, Trans. Amer. Math. Soc. (1995), to appear.

[12] J. P. Eckmann and D. Ruelle, Ergodic theory of chaos and strange attractors, Rev. Mod. Phys. 57 (1985), 617-656.

[13] J. P. Gauthier, I. Kupka and G. Sallet, Controllability of right invariant systems on real semi simple Lie groups, Systems Control Lett. 5 (1984), 187-190.

[14] W. Hahn, The Stability of Motion, Springer, 1967.

[15] E. C. Joseph, Stability radii of two dimensional bilinear systems, Ph. D. Thesis, Department of Mathematics, Iowa State, 1993.

[16] A. M. Lyapunov, Problème générale de la stabilité du mouvement, Comm. Soc. Math. Kharkov 2 (1892).

[17] V. I. Oseledeč, A multiplicative ergodic theorem. Lyapunov characteristic numbers for dynamical systems, Trans. Moscow Math. Soc 19 (1968), 197-231.

[18] E. D. Sontag, Feedback stabilization of nonlinear systems, in: Robust Control of Linear Systems and Nonlinear Control, M. A. Kashoek, J. H. van Schuppen, A. C. Ran (eds.) Birkhäuser, 1990, 61-81. 